\title{
$\mathrm{PdO}, \mathrm{Au}, \mathrm{CdO}$ 修饰 $\mathrm{SnO}_{2}$ 纳米纤维的制备及其气敏特性
}

\author{
胡瑞金 王 兢 ${ }^{*}$ 朱慧超 \\ (大连理工大学电子科学与技术学院, 辽宁大连 116023)
}

\begin{abstract}
摘要：采用静电纺丝的方法制备了 $\mathrm{SnO}_{2}$ 纳米纤维, 并分别用PdO、 $\mathrm{Au} 、 \mathrm{CdO}$ 对该纳米纤维材料进行表面修 饰. 用X射线衍射 (XRD)、扫描电子显微镜 (SEM)、X射线能谱(EDX)、X射线光电子能谱 (XPS)分析、 Brunauer-Emmett-Teller (BET)比表面积测试对材料进行表征. 修饰前后, $\mathrm{SnO}_{2}$ 纳米纤维都是由约 $15 \mathrm{~nm}$ 的纳 米颗粒构成的直径约为 $200 \mathrm{~nm}$ 的多级结构材料. 采用静态测试系统对纯 $\mathrm{SnO}_{2}$ 及不同物质修饰的 $\mathrm{SnO}_{2}$ 的气敏 特性进行测试, 结果表明, 未修饰的 $\mathrm{SnO}_{2}$ 纳米纤维气敏元件对甲醛具有较好的响应. 修饰后的 $\mathrm{SnO}_{2}$ 材料的气敏 特性都有明显的改善. $\mathrm{CdO}$ 修饰的 $\mathrm{SnO}_{2}$ 气敏元件对甲醛的响应值最高, 且响应恢复时间短, 选择性好. Au修饰 的 $\mathrm{SnO}_{2}$ 气敏元件对甲醛响应的最佳工作温度从 $300{ }^{\circ} \mathrm{C}$ 降到了 $200{ }^{\circ} \mathrm{C}$. 经 $\mathrm{PdO}$ 修饰后, $\mathrm{SnO}_{2}$ 纳米纤维对甲苯的 响应值变得最高. 初步分析了经过修饰的 $\mathrm{SnO}_{2}$ 气敏材料的敏感机理.
\end{abstract}

关键词: 气体传感器; $\mathrm{SnO}_{2}$; 修饰; 静电纺丝; 气敏机理

中图分类号: 0649

\section{Preparation and Gas Sensing Properties of PdO, Au, CdO Coatings on $\mathrm{SnO}_{2}$ Nanofibers}

\author{
HU Rui-Jin ～WANG Jing* ZHU Hui-Chao \\ (School of Electronic Science and Technology, Dalian University of Technology, Dalian 116023, Liaoning Province, P. R. China)
}

\begin{abstract}
SnO}_{2}$ nanofibers fabricated by electrospinning were coated with $\mathrm{PdO}, \mathrm{Au}$, and $\mathrm{CdO}$. X-ray diffraction (XRD), scanning electron microscopy (SEM), energy dispersive X-ray spectrometry (EDX), X-ray photoelectron spectroscopy (XPS), and Brunauer-Emmett-Teller (BET) tests were used to characterize the nanofibers. The diameters of bare and coated $\mathrm{SnO}_{2}$ nanofibers were approximately $200 \mathrm{~nm}$, and had 15-nm diameter grains. The gas-sensing properties of all the nanofibers were characterized under static gas conditions. The results indicated that the bare $\mathrm{SnO}_{2}$ nanofibers were sensitive to formaldehyde; however the sensitivity of the coated nanofibers was better. In particular, the $\mathrm{CdO}$-coated $\mathrm{SnO}_{2}$ exhibited the highest sensitivity to formaldehyde, the shortest response and recovery times, and good selectivity. The operating temperature of the Au-coated $\mathrm{SnO}_{2}$ decreased from 300 to $200{ }^{\circ} \mathrm{C}$, while the PdO-coated $\mathrm{SnO}_{2}$ exhibited the highest sensitivity to toluene. The sensing mechanism of the coated $\mathrm{SnO}_{2}$ nanofibers was investigated.
\end{abstract}

Key Words: Gas sensor; $\mathrm{SnO}_{2}$; Coating; Electrospinning; Gas sensing mechanism

1 引言

半导体材料 1,2 有着较大的比表面积, 且其价格
低廉、制备工艺简单, 其检测的气体浓度可以达到 体积分数为 $10^{-6}$ 量级, 因此其在气体检测领域有着

Received: May 25, 2015; Revised: August 21, 2015; Published on Web: August 24, 2015.

*Corresponding author. Email: wangjing@dlut.edu.cn; Tel: +86-411-84708382; Fax: +86-411-84706706.

The project was supported by the National Natural Science Foundation of China $(61176068,61131004,61107028)$.

国家自然科学基金(61176068, 61131004, 61107028)资助项目

CEditorial office of Acta Physico-Chimica Sinica 
重要的应用. 零维半导体纳米材料即纳米颗粒材料 容易发生团聚, 导致材料的气敏特性降低, 同时也 不利于材料的长期稳定性. 而一维材料 ${ }^{3}$ 的出现则改 善了团聚的现象. 同时, 一维材料比如纳米线、纳 米纤维等形成网状支架结构, 也增强了材料的通透 性. 除了改善材料的微观结构外, 对已知的材料做 进一步的处理也是改善材料气敏特性行之有效的 方法, 常见的处理方法是将多种材料进行复合, 对 材料进行掺杂和修饰.

由于不同材料的特性不同, 将多种材料进行复 合可以实现优势互补, 增强材料的气敏特性. Khoang 等 ${ }^{4}$ 结合热蒸发和水热两种方法将 $\mathrm{SnO}_{2}$ 和 $\mathrm{ZnO}$ 复合, 制备出刷子结构的纳米材料, 其对体积分数为 $100 \times$ $10^{-6}$ 乙醇的响应值比纯 $\mathrm{SnO}_{2}$ 高3 倍. $\mathrm{Lin}^{\text {等 }}{ }^{5}$ 用水热法 制备了 $\mathrm{SnO}_{2}$ 与还原石墨烯的复合材料, 实现了室温 下对 $\mathrm{NH}_{3}$ 的检测. 掺杂为一种最常见的提高气敏特 性的方法, 掺杂即为在材料的制备过程中加入金属 盐, 金属离子在材料中形成替位或者间隙掺杂, 从 而影响材料的特性. 金属氧化物 $\mathrm{CuO} 、{ }^{6} \mathrm{CeO} 、{ }^{7}$ $\mathrm{MoO}_{3} 、{ }^{8} \mathrm{ZnO} 、{ }^{9} \mathrm{NiO}^{10,11}$ 等都可作为掺杂物或复合 物. Sun等研究了不同浓度 $\mathrm{Cu}$ 掺杂的 $\mathrm{Fe}_{2} \mathrm{O}_{3}$ 对乙醇的 响应, 结果表明 $3 \%$ ( $w$, 质量分数) $\mathrm{Cu}$ 掺杂材料的气 敏响应最好, 对体积分数为 $100 \times 10^{-6}$ 乙醇的响应值 是纯 $\mathrm{Fe}_{2} \mathrm{O}_{3}$ 的近3 倍.

而常见的半导体气敏材料如 $\mathrm{SnO}_{2} 、{ }^{12} \mathrm{ZnO} 、{ }^{13}$ $\mathrm{In}_{2} \mathrm{O}_{3} 、{ }^{14} \mathrm{TiO}_{2}{ }^{15}$ 等为表面控制型材料, 即被测气体与 气敏材料的相互作用只发生在材料的表层, 这样材 料的气敏特性就与材料的表面特性息息相关,而修 饰则是改善材料表面特性的一种方法. 表面修饰的 修饰物既有普通金属/半导体氧化物, 如 $\mathrm{CuO} 、{ }^{16}$ $\mathrm{Cr}_{2} \mathrm{O}_{3} 、{ }^{17} \mathrm{La}_{2} \mathrm{O}_{3} 、{ }^{18} \mathrm{Fe}_{2} \mathrm{O}_{3} 、{ }^{19} \mathrm{NiO},{ }^{20}$ 也有如 $\mathrm{Au} 、{ }^{21}$ $\mathrm{Pd} 、{ }^{22} \mathrm{Pt} 、{ }^{23} \mathrm{Ag}^{24}$ 等贵金属. 修饰的方法也多种多样, 如溅射法、水热合成法、一釜合成法、浸渍法等. $\mathrm{Liu}^{\text {等 }}{ }^{25}$ 在 $\mathrm{WO}_{3}$ 上修饰了 $\mathrm{Pt}$ 纳米颗粒, 用于检测乙醇. 修饰后 $\mathrm{WO}_{3}$ 的最佳工作温度降低为 $180^{\circ} \mathrm{C}$, 且其相 应时间从修饰前的 $39 \mathrm{~s}$ 减少为 $13 \mathrm{~s}$. 修饰前后 $\mathrm{WO}_{3}$ 对 体积分数为 $100 \times 10^{-6}$ 乙醇的响应值由 2.6 提高为 5.8 . 对半导体材料进行不同物质的修饰可以达到提高 材料气敏特性的效果, 其原因可以从以下几个方面 来解释, 首先, 贵金属材料对还原性气体的氧化有 催化作用, 可以使还原性气体与氧离子的反应可以 在较低的温度下发生; 其次, 半导体材料进行修饰
后会产生溢流效应; 再次, 金属修饰于半导体材料 表面可能会形成肖特基接触, 加宽半导体材料的耗 尽层.

本文采用静电纺丝法制备了 $\mathrm{SnO}_{2}$ 纳米纤维, 分 别以 $\mathrm{PdO} 、 \mathrm{Au} 、 \mathrm{CdO}$ 为修饰物, 用浸渍法和溅射法 对 $\mathrm{SnO}_{2}$ 纳米纤维进行修饰改性. 采用 $\mathrm{X}$ 射线衍射 $(X R D)$ 、扫描电子显微镜 $(S E M) 、 X$ 射线能谱 $(E D X) 、 X$ 射线光电子能谱(XPS)分析、BrunauerEmmett-Teller (BET)比表面积测试等表征手段对材 料的形貌、组分进行表征, 采用静态测试系统对修 饰前后的 $\mathrm{SnO}_{2}$ 进行气敏测试, 并对材料气敏机理进 行简要分析.

\section{2 实验部分}

\section{1 材料制备及表征}

聚乙烯吡咯烷酮 (PVP) 购于美国 Sig m a Aldrich公司, 氯化亚锡 $\left(\mathrm{SnCl}_{2} \cdot 2 \mathrm{H}_{2} \mathrm{O}\right) 、 \mathrm{~N}, \mathrm{~N}$-二甲基 四酰胺 $(\mathrm{DMF}) 、$ 无水乙醇 $(99.0 \%)$ 购于国药集团化 学试剂(中国). 上述化学试剂均为分析纯, 未经进一 步纯化.

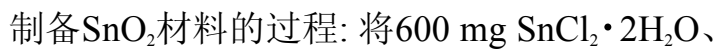
$600 \mathrm{mg}$ PVP溶于 $3 \mathrm{~mL}$ DMF、 $4 \mathrm{~mL}$ 乙醇的混合溶液 中, 在磁力搅拌器上搅拌 $8 \mathrm{~h}$ 形成透明均匀的粘稠纺 丝前驱液. 将此前驱液倒入装有 7 号针头的针筒中, 在针头和收集铜板间施加电压为 $20 \mathrm{kV}$ 的静电场, 针头和收集板间距离为 $15 \mathrm{~cm}$. 在重力和电场力的 作用下, 前驱液形成喷射细流落到收集板上. 在 600 ${ }^{\circ} \mathrm{C}$, 空气气氛中烧结 $2 \mathrm{~h}$, 即得到 $\mathrm{SnO}_{2}$ 纳米纤维.

$\mathrm{PdO}$ 修饰 $\mathrm{SnO}_{2}$ 的过程如下: 量取 $10 \mathrm{~mL}$ 甲醇, 放 入 $3 \mathrm{mg}$ 的 $\mathrm{PdCl}_{2}$, 对该混合液进行超声及搅拌处理, 使 $\mathrm{PdCl}_{2}$ 溶解. 在该溶液中加入 $60 \mathrm{mg}$ 已经制备好的 $\mathrm{SnO}_{2}$ 纳米纤维, 用磁力搅拌器缓慢搅拌 $30 \mathrm{~min}$. 将得 到的沉淀物在 $80^{\circ} \mathrm{C}$ 下干燥 $3 \mathrm{~h}$, 用马弗炉在 $600{ }^{\circ} \mathrm{C}$ 下 烧结 $2 \mathrm{~h}$, 最终得到了 $\mathrm{PdO}$ 修饰的 $\mathrm{SnO}_{2}$.

$\mathrm{Au}$ 修饰 $\mathrm{SnO}_{2}$ 过程如下: 将涂有 $\mathrm{SnO}_{2}$ 纳米纤维粉 末的玻璃片放入溅射室内, 在 $5 \mathrm{~mA}$, 真空度 $0.1 \mathrm{~Pa}$ 的 条件下, 溅射 $\mathrm{Au} 20 \mathrm{~s}$. 将获得的材料在马弗炉中 300 ${ }^{\circ} \mathrm{C}$ 下退火 $1 \mathrm{~h}$.

$\mathrm{CdO}$ 修饰 $\mathrm{SnO}_{2}$ 过程如下: 称取 $46.3 \mathrm{mg}$ 的 $\mathrm{Cd}\left(\mathrm{NO}_{3}\right)_{2} \cdot 4 \mathrm{H}_{2} \mathrm{O}$ 溶于 $5 \mathrm{~mL}$ 乙醇中, 搅拌 $2 \mathrm{~h}$, 形成透明 均匀溶液. 将 $60 \mathrm{mg} \mathrm{SnO}_{2}$ 纳米纤维粉末加入溶液中, 缓慢搅拌 $20 \mathrm{~min}, 80^{\circ} \mathrm{C}$ 下干燥 $3 \mathrm{~h}, 500^{\circ} \mathrm{C}$ 下空气气 
氛中烧结 $2 \mathrm{~h}$, 即得到 $\mathrm{CdO}$ 修饰 $\mathrm{SnO}_{2}$ 纳米纤维.

材料的形貌和组分用 $X$ 射线衍射、场发射扫描 电镜(FE-SEM)、X射线能谱仪、比表面积和孔径分 析仪、 $X$ 射线光电子能谱仪分析. 所用 $X$ 射线粉末衍 射仪型号为 D/Max 2400 (Rigaku, Japan), 扫描角度 为 $20^{\circ}-80^{\circ}$, 扫描速率为 $10\left(^{\circ}\right) \cdot \mathrm{min}^{-1}$. 场发射扫描电 镜的型号为NOVA NanoSEM 150 (American). 比表 面积和孔径分析仪的型号为AUTOSORB-1-MP (American). X射线光电子能谱分析仪型号为 ESCALAB 250Xi (American).

\section{2 元件的制作与测量}

分别将修饰前后的 $\mathrm{SnO}_{2}$ 与去离子水混合, 放入 研钵中研磨 $30 \mathrm{~min}$, 形成糊状物, 将其均匀地涂到带 有金电极的氧化铝陶瓷管上. 将涂好的元件放入马 弗炉中在 $300{ }^{\circ} \mathrm{C}$ 下烧结 $2 \mathrm{~h}$. 在烧结好的元件中间插 入Ni-Cr合金加热丝，并将其焊接到六角基座上，制 成旁热式气敏元件, 将元件放在老化台上老化一周 后进行测量.

元件气敏特性的测量采用静态测试系统. 该测 试系统由测试腔、测试电路、数据采集卡、上位 机显示系统组成. 测试腔为 $50 \mathrm{~L}$ 的密封聚四氟乙烯 箱, 测试气体通过有机液体汽化的方式扩散到整个 测试箱. 注入测试箱的液体体积 $(V)$ 与气体浓度 (C) 的关系为如式(1)所示:

$$
\rho V \omega / M=C \times 50 /(22.4 \times(273+T) / 273)
$$

其中 $\rho$ 为液体密度, $\omega$ 为液体质量分数, $M$ 为被测气 体的摩尔质量, $T$ 为测试箱内温度.

测试电路为一个分压电路, 即通过检测气敏元 件在空气中的分压 $V_{\text {air }}$ 和被测气体中的分压 $V_{\text {gas }}$ 从而 得到气敏元件电阻变化, 进而计算出气敏元件的灵 敏度. 该电路的总电压为 $10 \mathrm{~V}$, 外接电阻值为 $R_{\mathrm{L}}$. 灵 敏度 $(S)$ 为气敏元件在空气中的电阻值 $R_{\mathrm{a}}$ 和被测气 体中的电阻值 $R_{\mathrm{g}}$ 之比:

$$
\begin{aligned}
& S=\frac{R_{\mathrm{a}}}{R_{\mathrm{g}}} \\
& R_{\mathrm{a}}=R_{\mathrm{L}} \times V_{\text {air }} /\left(10-V_{\text {air }}\right) \\
& R_{\mathrm{a}}=R_{\mathrm{L}} \times V_{\text {gas }} /\left(10-V_{\text {gas }}\right)
\end{aligned}
$$

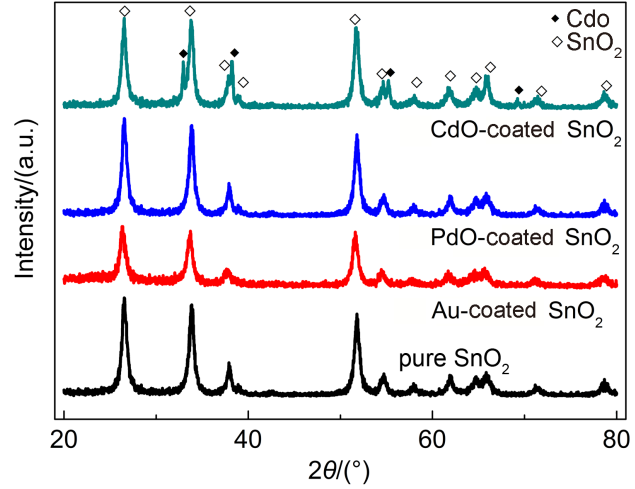

图1 纯 $\mathrm{SnO}_{2}$ 和不同物质修饰的 $\mathrm{SnO}_{2}$ 的XRD图谱

Fig.1 XRD patterns of pure $\mathrm{SnO}_{2}$ and different substances coated $\mathrm{SnO}_{2}$

\section{3 结果与讨论}

\section{1 材料的表征}

图1为纯 $\mathrm{SnO}_{2}$ 和不同物质修饰 $\mathrm{SnO}_{2}$ 的 XRD图谱, 可以看出, $\mathrm{CdO}$ 修饰 $\mathrm{SnO}_{2}$ 材料中有修饰物 $\mathrm{CdO}$ 的衍 射峰(对应标准卡片PDF 05-0640). 而PdO和Au修饰 的 $\mathrm{SnO}_{2}$ 材料中却没有明显的修饰物的峰. 表 1 中给 出了各材料的平均粒径, 除 $\mathrm{CdO}$ 修饰的 $\mathrm{SnO}_{2}$ 材料的 平均粒径相对于纯 $\mathrm{SnO}_{2}$ 的平均粒径有所增大外, $\mathrm{Au} 、 \mathrm{PdO}$ 修饰的 $\mathrm{SnO}_{2}$ 材料的平均粒径都有所减小.

图2为纯 $\mathrm{SnO}_{2}$ 和 $\mathrm{PdO} 、 \mathrm{Au} 、 \mathrm{CdO}$ 修饰的 $\mathrm{SnO}_{2}$ 材 料的SEM图. 从图中可以看出, 修饰前后材料均为 纳米纤维状, 表面有很多可见的孔洞, 纳米纤维直 径都在 $200 \mathrm{~nm}$ 左右. $\mathrm{Au}$ 修饰的 $\mathrm{SnO}_{2}$ 纳米纤维表面, 除了 $15 \mathrm{~nm}$ 左右的 $\mathrm{SnO}_{2}$ 颗粒外, 还有 $7 \mathrm{~nm}$ 左右的 $\mathrm{Au}$ 粒子, 如图 2(c)中白色箭头所指. $\mathrm{CdO}$ 修饰的 $\mathrm{SnO}_{2}$ 纳米纤维表面粘连了一些纳米团装物, 如图 2(d)中白色箭头所指, 可能为 $\mathrm{CdO}$ 颗粒; 而 $\mathrm{PdO}$ 修饰 的 $\mathrm{SnO}_{2}$ 纳米纤维不能明显的看出修饰的 $\mathrm{PdO}$ 粒子, 可能是由于 $\mathrm{PdO}$ 粒子大小与组成 $\mathrm{SnO}_{2}$ 的小颗粒大小 相近, 故而淹没在 $\mathrm{SnO}_{2}$ 的小颗粒中, 无法直观的看 出. 图3给出纯 $\mathrm{SnO}_{2}$ 和不同物质修饰 $\mathrm{SnO}_{2}$ 纤维的 EDX图. 从图中谱线可以清楚地看到Pd元素的存在.

对纯 $\mathrm{SnO}_{2}$ 和 $\mathrm{PdO}$ 修饰的 $\mathrm{SnO}_{2}$ 进行BET测试分析, 其氮气吸附脱附曲线和孔分布曲线分别如图 4(a, b)所示. 从图4(a)可以看出, 纯 $\mathrm{SnO}_{2}$ 和 $\mathrm{PdO}$ 修饰的

表1 纯 $\mathrm{SnO}_{2}$ 和不同物质修饰 $\mathrm{SnO}_{2}$ 的平均粒径

Table 1 Average crystallite sizes of pure $\mathrm{SnO}_{2}$ and different substances coated $\mathrm{SnO}_{2}$

\begin{tabular}{ccccc}
\hline Material type & pure $\mathrm{SnO}_{2}$ & Au-coated $\mathrm{SnO}_{2}$ & PdO-coated $\mathrm{SnO}_{2}$ & $\mathrm{CdO}_{\text {-coated } \mathrm{SnO}_{2}}$ \\
\hline Average grain size/nm & 14.8 & 12.3 & 12.7 & 16.5 \\
\hline
\end{tabular}



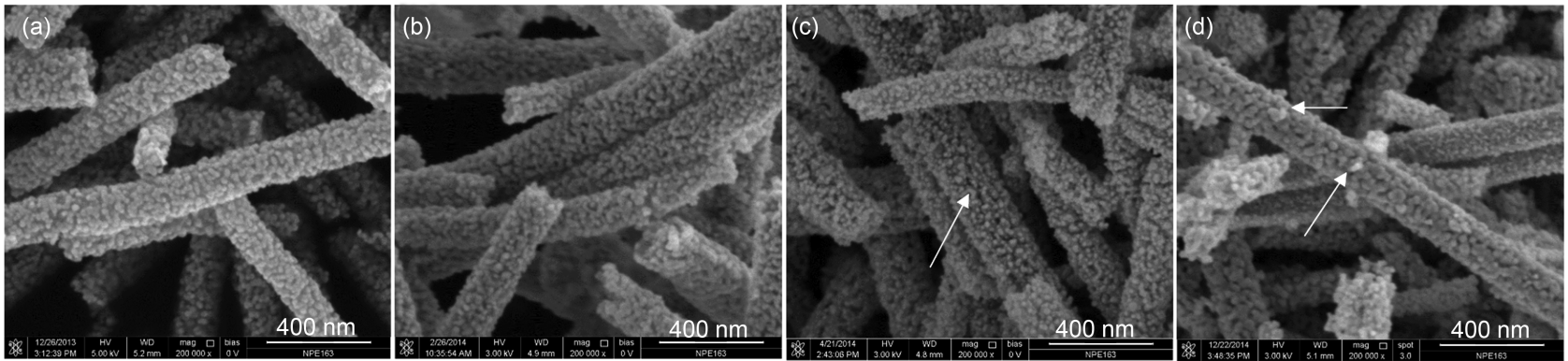

图2 纯 $\mathrm{SnO}_{2}$ 和不同物质修饰 $\mathrm{SnO}_{2}$ 纳米纤维的 $\mathrm{SEM}$ 图

Fig.2 SEM images of pure $\mathrm{SnO}_{2}$ and different substances coated $\mathrm{SnO}_{2}$ nanofibers

(a) pare $\mathrm{SnO}_{2}$, (b) $\mathrm{PdO}$-coated $\mathrm{SnO}_{2}$, (c) Au-coated $\mathrm{SnO}_{2}$, (d) CdO-coated $\mathrm{SnO}_{2}$

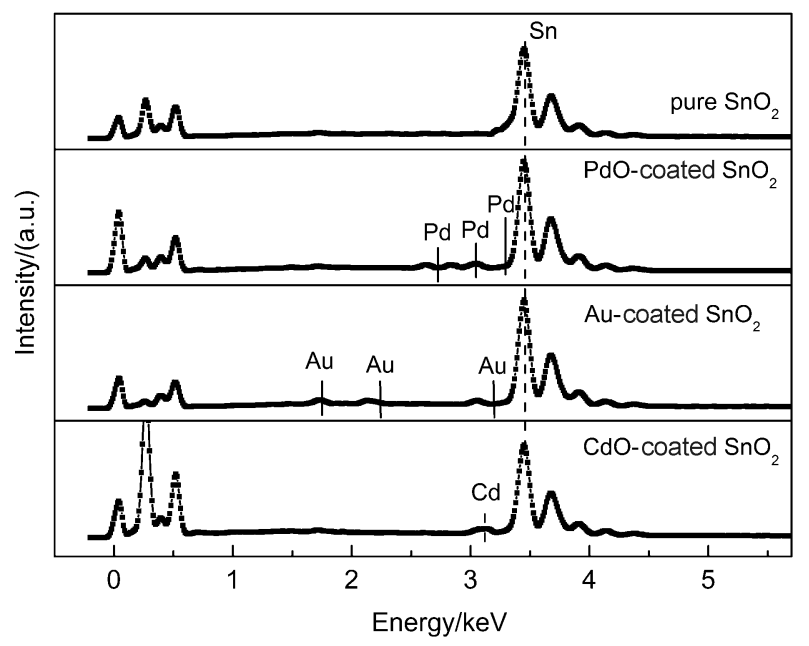

图3 纯 $\mathrm{SnO}_{2}$ 和不同物质修饰的 $\mathrm{SnO}_{2}$ 的EDX图

Fig.3 EDX spectra of pure $\mathrm{SnO}_{2}$ and different substances coated $\mathrm{SnO}_{2}$

$\mathrm{SnO}_{2}$ 的氮气吸附脱附曲线均为 IV 型回滞曲线, 具有 H3 型滞后环. IV型回滞曲线说明液氮在材料中产生 毛细管冷凝现象, $\mathrm{H} 3$ 型滞后环表明纯 $\mathrm{SnO}_{2}$ 和 $\mathrm{PdO}$ 修
饰的 $\mathrm{SnO}_{2}$ 的介孔是由于粒子堆积形成的细长介孔 和管道.

纯 $\mathrm{SnO}_{2}$ 和 $\mathrm{PdO}$ 修饰的 $\mathrm{SnO}_{2}$ 的比表面积、孔分布 和孔容等数据列于表 2 , 纯 $\mathrm{SnO}_{2}$ 的比表面积为 37.26 $\mathrm{m}^{2} \cdot \mathrm{g}^{-1}$, 修饰 $\mathrm{PdO}$ 后比表面积增为 $52.15 \mathrm{~m}^{2} \cdot \mathrm{g}^{-1}$, 说明 修饰 $\mathrm{PdO}$ 后材料的比表面积有较为明显的增加. 纯 $\mathrm{SnO}_{2}$ 的介孔分布在10-17 $\mathrm{nm}$ 之间，而PdO修饰后的 $\mathrm{SnO}_{2}$ 的介孔分布在5-8 $\mathrm{nm}$ 之间, 推测是由于有部分 表面修饰的 $\mathrm{PdO}$ 粒子堆积在了原 $\mathrm{SnO}_{2}$ 的介孔上从而 导致材料的介孔尺寸有所减小. $\mathrm{PdO}$ 修饰后的 $\mathrm{SnO}_{2}$ 的孔容也有所减小.

对纯 $\mathrm{SnO}_{2}$ 和修饰 $\mathrm{Au}$ 后 $\mathrm{SnO}_{2}$ 材料进行XPS表征, 图5给出 $\mathrm{Sn} 3 d$ 谱线图. 从图中看出, 修饰 $\mathrm{Au}$ 后 $\mathrm{SnO}_{2}$ 材料的 $\mathrm{Sn}$ 峰向高结合能方向移动, 退火温度为 300 ${ }^{\circ} \mathrm{C}$ 的 $\mathrm{Au}$ 修饰 $\mathrm{SnO}_{2}$ 的 $\mathrm{Sn}$ 峰移动了 $0.65 \mathrm{eV}$. 而 $\mathrm{Sn}$ 峰位 的移动是由于 $\mathrm{Au}$ 和 $\mathrm{SnO}_{2}$ 的接触, $\mathrm{Au}$ 从 $\mathrm{SnO}_{2}$ 表面夺 取电子使得Sn峰向高结合能方向移动.

图6给出了纯 $\mathrm{SnO}_{2}$ 和修饰 $\mathrm{Au}$ 后 $\mathrm{SnO}_{2}$ 材料 $\mathrm{O} 1 s$ 的
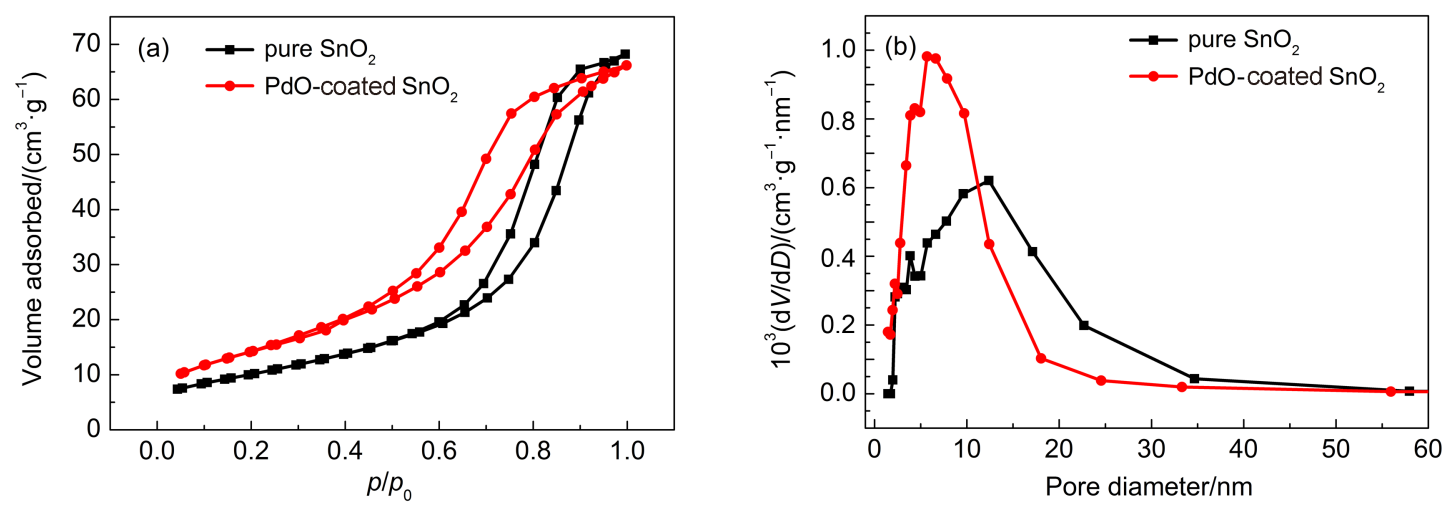

图4 纯 $\mathrm{SnO}_{2}$ 和PdO修饰 $\mathrm{SnO}_{2}$ 的(a)氮气吸附-脱附等温曲线及(b)对应的孔径分布曲线

Fig.4 (a) Nitrogen adsorption-desorption isothermal curves and (b) the corresponding pore size distributions of pure $\mathrm{SnO}_{2}$ and PdO-coated $\mathrm{SnO}_{2}$ 
表2 纯 $\mathrm{SnO}_{2}$ 和PdO修饰的 $\mathrm{SnO}_{2}$ 的比表面积、孔分布和孔容

Table 2 Specific surface area, pore size distribution, and specific volume of pure and PdO-coated $\mathrm{SnO}_{2}$

\begin{tabular}{cccc}
\hline Material type & Specific surface area $/\left(\mathrm{m}^{2} \cdot \mathrm{g}^{-1}\right)$ & Pore size distribution $/ \mathrm{nm}$ & Pore volume $/\left(\mathrm{cm}^{3} \cdot \mathrm{g}^{-1}\right)$ \\
\hline pure $\mathrm{SnO}_{2}$ & 37.26 & $10-17$ & 0.1055 \\
PdO-coated $\mathrm{SnO}_{2}$ & 52.15 & $5-8$ & 0.1024 \\
\hline
\end{tabular}

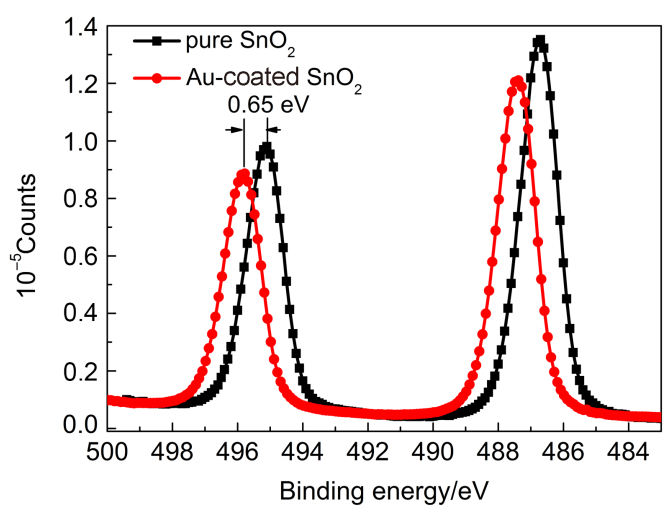

图5 纯 $\mathrm{SnO}_{2}$ 和 $\mathrm{Au}$ 修饰 $\mathrm{SnO}_{2}$ 的 $\mathrm{Sn} 3 \mathrm{dXPS}$ 图

Fig.5 Sn 3d XPS spectra of pure and Au-coated $\mathrm{SnO}_{2}$

XPS谱图, 并进行分峰拟合. 纯 $\mathrm{SnO}_{2}$ 结合能分别为 530.6 和 $531.5 \mathrm{eV}$, 分别为晶格氧和吸附氧. 计算得到 纯 $\mathrm{SnO}_{2}$ 和 $\mathrm{Au}$ 修饰 $\mathrm{SnO}_{2}$ 的吸附氧和 $\mathrm{Sn}$ 的比分别为 0.58 和 $0.66, \mathrm{Au}$ 修饰后 $\mathrm{SnO}_{2}$ 材料的 $\mathrm{O}_{\text {adsorbed }} / \mathrm{Sn}$ 比增大, 说明 $\mathrm{Au}$ 修饰后, $\mathrm{SnO}_{2}$ 对氧的吸附能力增加.

\section{2 气敏特性}

图7给出了纯 $\mathrm{SnO}_{2}$ 和不同物质修饰 $\mathrm{SnO}_{2}$ 材料的 最佳工作温度测试曲线, 图7(a)中纯 $\mathrm{SnO}_{2}$ 和PdO修饰 的 $\mathrm{SnO}_{2}$ 其测试气体为 $10 \times 10^{-6}$ (体积分数)甲苯, 图 7(b)中纯 $\mathrm{SnO}_{2}$ 和 $\mathrm{Au} 、 \mathrm{CdO}$ 修饰的 $\mathrm{SnO}_{2}$ 其测试气体为 $10 \times 10^{-6}$ (体积分数)甲醛. 图 8 为纯 $\mathrm{SnO}_{2}$ 和不同物质 修饰 $\mathrm{SnO}_{2}$ 对不同浓度甲苯或者甲醛的响应曲线.

从图7可以看出, 修饰前, $\mathrm{SnO}_{2}$ 纳米纤维气敏元

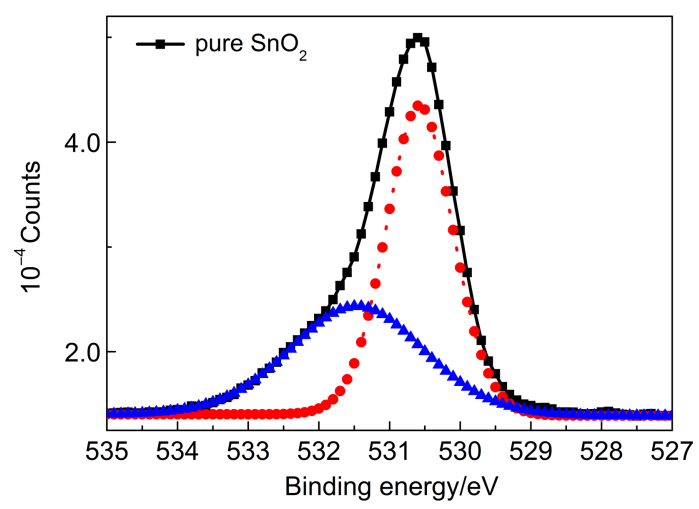

件的最佳工作温度为 $300{ }^{\circ} \mathrm{C}, \mathrm{Au}$ 修饰的 $\mathrm{SnO}_{2}$ 气敏元 件对甲醛的最佳工作温度降到 $200{ }^{\circ} \mathrm{C}$. 图7和图 8 都 表明, 修饰后 $\mathrm{SnO}_{2}$ 对甲苯和甲醛的响应值都提高了. 未修饰和 $\mathrm{CdO}$ 修饰 $\mathrm{SnO}_{2}$ 气敏元件对 $10 \times 10^{-6}$ 甲醛的 响应值分别为 5.6和 23.4, 响应值有了明显提高. 而 $\mathrm{PdO}$ 修饰的 $\mathrm{SnO}_{2}$ 纳米纤维气敏元件出现对甲苯的响 应值变得最高的现象.

选择性是半导体气体传感器的重要性能之一. 图9为纯 $\mathrm{SnO}_{2}$ 和不同物质修饰 $\mathrm{SnO}_{2}$ 材料对 $10 \times 10^{-6}$ (体积分数)甲醛、乙醇、甲醇、甲苯、氨气的响应 值. 从图中看出, 纯 $\mathrm{SnO}_{2}$ 气敏元件对甲醛的气敏响 应值高于对其它气体, 为 5.6. 虽然纯 $\mathrm{SnO}_{2}$ 气敏元件 对甲醛的响应高于对其它几种气体, 但差别并不明 显. $\mathrm{Au}$ 修饰的 $\mathrm{SnO}_{2}$ 气敏元件对 $10 \times 10^{-6}$ 甲醛的响应 值为 8.96 , 也高于对其它气体, 但差别仍不明显. $\mathrm{CdO}$ 修饰 $\mathrm{SnO}_{2}$ 气敏元件对 $10 \times 10^{-6}$ 甲醛的响应值为 23.4, 分别是对乙醇和丙酮响应值的 2.7 倍和 4.3 倍, 甲醛和其他气体响应值差别最大, 表明 $\mathrm{CdO}$ 修饰对 $\mathrm{SnO}_{2}$ 气敏元件选择性的改善最明显.

$\mathrm{PdO}$ 修饰 $\mathrm{SnO}_{2}$ 气敏元件出现另一种现象, 对甲 苯的响应值变大, 对甲醛的响应值反而有所降低.

表3给出不同物质修饰 $\mathrm{SnO}_{2}$ 的各项气敏特性比 较, 可以看出纯 $\mathrm{SnO}_{2}$ 气敏元件对 $10 \times 10^{-6}$ 甲醛的响 应恢复时间分别为 20 和 $37 \mathrm{~s}, \mathrm{Au}$ 修饰 $\mathrm{SnO}_{2}$ 气敏元件 的响应恢复时间比纯 $\mathrm{SnO}_{2}$ 气敏元件略有增长,

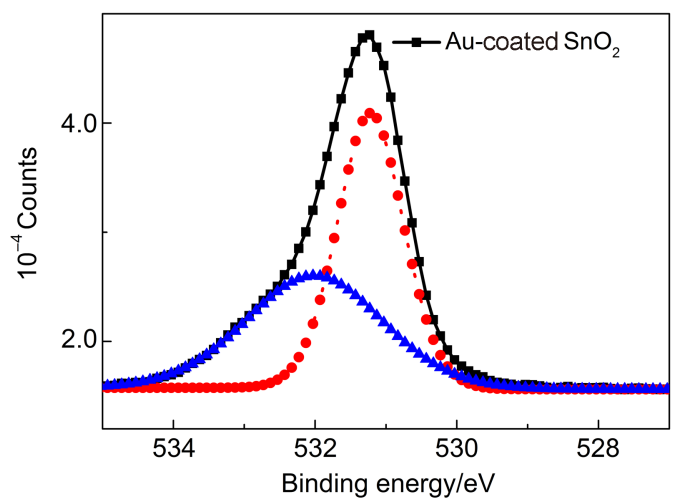

图6 纯 $\mathrm{SnO}_{2}$ 和 $\mathrm{Au}$ 修饰 $\mathrm{SnO}_{2}$ 的 $\mathrm{O} 1 \mathrm{~s} \mathrm{XPS}$ 图

Fig.6 O $1 s$ XPS spectra of pure and Au-coated $\mathrm{SnO}_{2}$ 

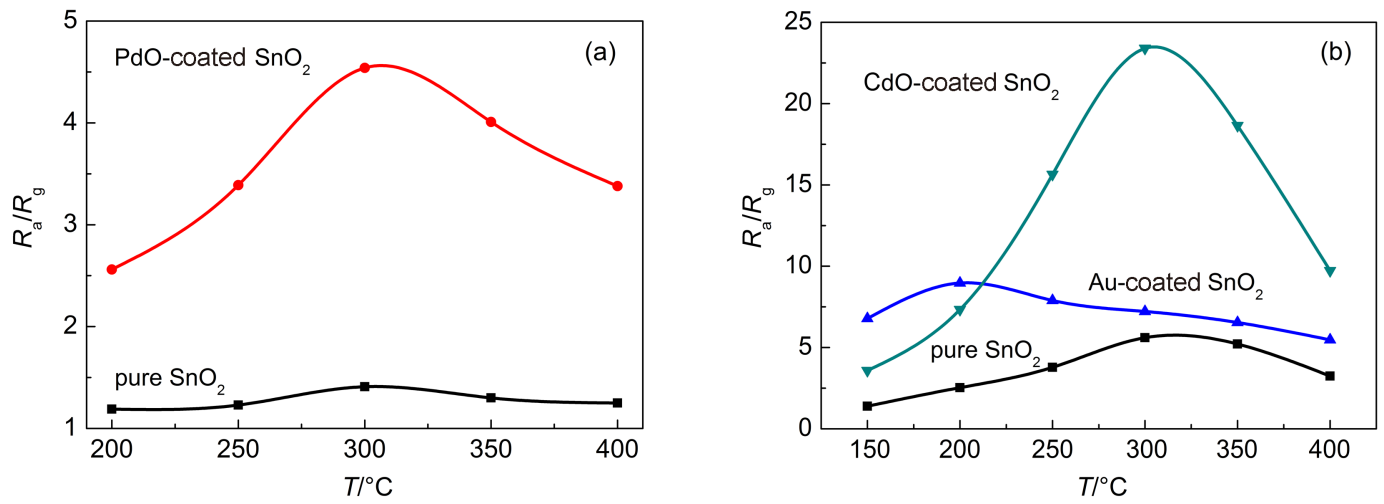

图7 不同工作温度下各气敏元件对 $10 \times 10^{-6}($ 体积分数 $)$ 气体的响应 $\left(\boldsymbol{R}_{\mathrm{a}} / \boldsymbol{R}_{\mathrm{g}}\right)$

Fig.7 Responses $\left(R_{\mathrm{a}} / R_{\mathrm{g}}\right)$ of sensors to $10 \times 10^{-6}$ (volume fraction) gases at different working temperatures testing gas: (a) toluene, (b) formaldehyde
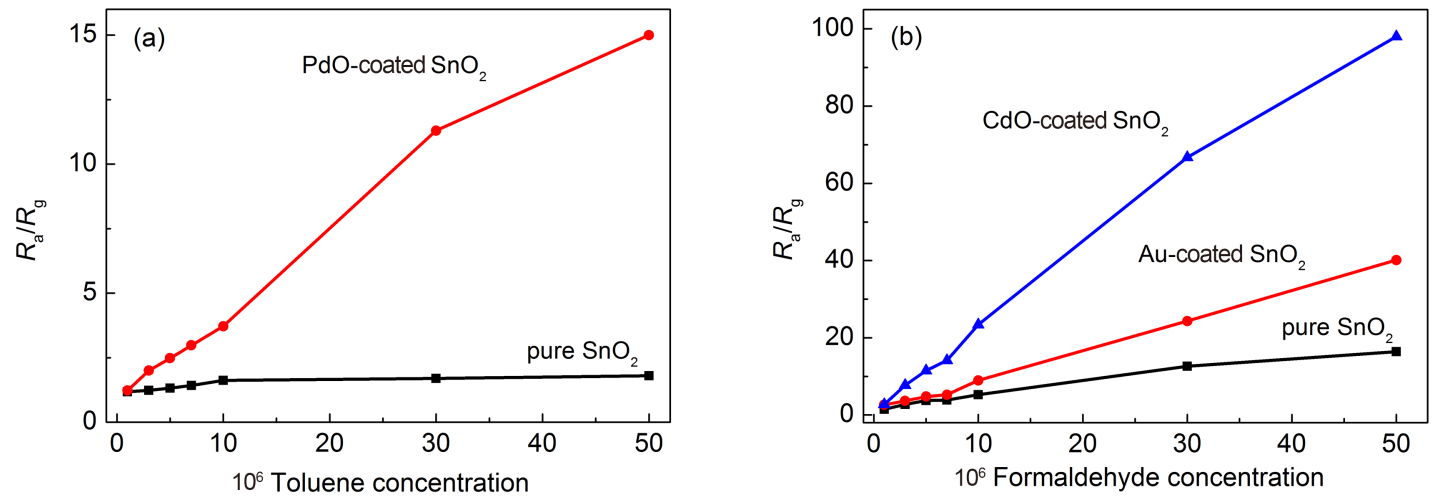

图8 纯 $\mathrm{SnO}_{2}$ 和不同物质修饰的气敏元件对不同浓度(体积分数)测试气体(a)甲苯、(b)甲醛的响应

Fig.8 Responses of pure and different substances coated $\mathrm{SnO}_{2}$ sensors to different concentrations (volume fraction) of testing gases of (a) toluene and (b) formaldehyde

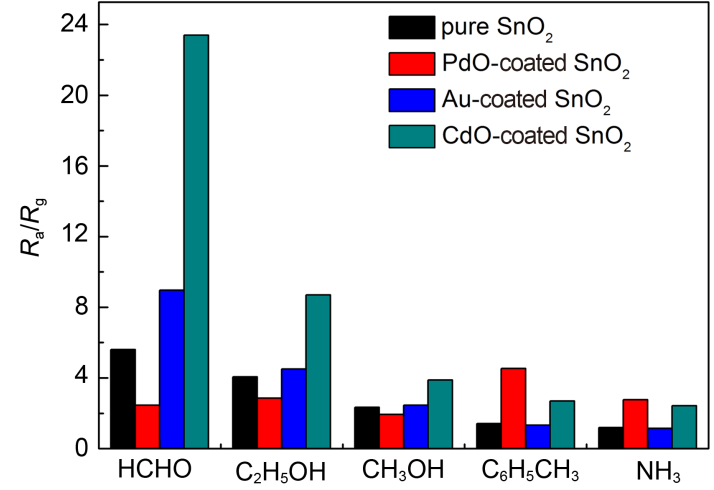

图9 气敏元件对 $10 \times 10^{-6}$ 不同气体的响应曲线

Fig.9 Response curves of sensors to $10 \times 10^{-6}$ different gases

$\mathrm{CdO}$ 修饰 $\mathrm{SnO}_{2}$ 气敏元件的响应恢复时间则有所减 短. $\mathrm{PdO}$ 修饰的 $\mathrm{SnO}_{2}$ 气敏元件对 $10 \times 10^{-6}$ 甲苯的响应 恢复时间虽然相对较长, 但对比纯 $\mathrm{SnO}_{2}$ 气敏元件对 甲苯的响应恢复时间 $120 \mathrm{~s} / 136 \mathrm{~s}$ 来说, 仍旧是有所 减小.

\section{3 敏感机理分析}

以 $\mathrm{PdO}$ 修饰的 $\mathrm{SnO}_{2}$ 为例分析修饰后 $\mathrm{SnO}_{2}$ 材料的 气体敏感机理. $\mathrm{SnO}_{2}$ 是一种 $n$ 型的表面控制型半导 体氧化物, 即 $\mathrm{SnO}_{2}$ 材料与还原性气体的反应基本是 发生在材料表面. 在空气中, $\mathrm{O}_{2}$ 吸附在材料表面, 从 材料中俘获电子形成 $\mathrm{O}_{2}^{-} 、 \mathrm{O}^{-} 、 \mathrm{O}_{2}^{-}$, 如式(5)-(8)所 示, 使材料电阻增大. 当环境温度低于 $100{ }^{\circ} \mathrm{C}$ 时氧离 子主要以 $\mathrm{O}_{2}^{-}$的形态存在, 当环境温度在 100 到 300 ${ }^{\circ} \mathrm{C}$ 时, 氧离子主要以 $\mathrm{O}^{-}$的形态存在, 当温度高于 300 ${ }^{\circ} \mathrm{C}$ 时, 氧离子主要以 $\mathrm{O}_{2}^{-}$的形态存在. 当材料暴露在 甲苯气体中时, 甲苯气体会与吸附在材料表面的氧 离子发生反应, 如式(9)及(10)所示, ${ }^{26}$ 这两个反应可 能单独发生也可能同时发生, 即产物可能是苯甲醛 或者是 $\mathrm{CO}_{2}$ 和 $\mathrm{H}_{2} \mathrm{O}$. 从而将捕获的电子重新释放回材 料, 使材料电阻减小. 因此材料的气敏特性与材料 表面吸附的氧离子的数量有很大的关系.

$\mathrm{O}_{2(\text { gas })} \rightarrow \mathrm{O}_{2(\text { ads })}$ 
表3 纯 $\mathrm{SnO}_{2}$ 和不同物质修饰 $\mathrm{SnO}_{2}$ 的气敏特性

Table 3 Gas sensing properties of pure $\mathrm{SnO}_{2}$ and different substances coated $\mathrm{SnO}_{2}$

\begin{tabular}{ccccc}
\hline Material type & Optimum operating temperature $/{ }^{\circ} \mathrm{C}$ & Highest response gas & Response to $10 \times 10^{-6}$ gas & Response $/$ recovery time/s \\
\hline pure $\mathrm{SnO}_{2}$ & 300 & formaldehyde & 5.60 & 8.96 \\
$\mathrm{Au}$-coated $\mathrm{SnO}_{2}$ & 200 & formaldehyde & $37 / 37$ \\
$\mathrm{PdO}$-coated $\mathrm{SnO}_{2}$ & 300 & toluene & 4.54 & 23.4 \\
$\mathrm{CdO}$-coated $\mathrm{SnO}_{2}$ & 300 & formaldehyde & $20 / 63$ \\
\hline
\end{tabular}

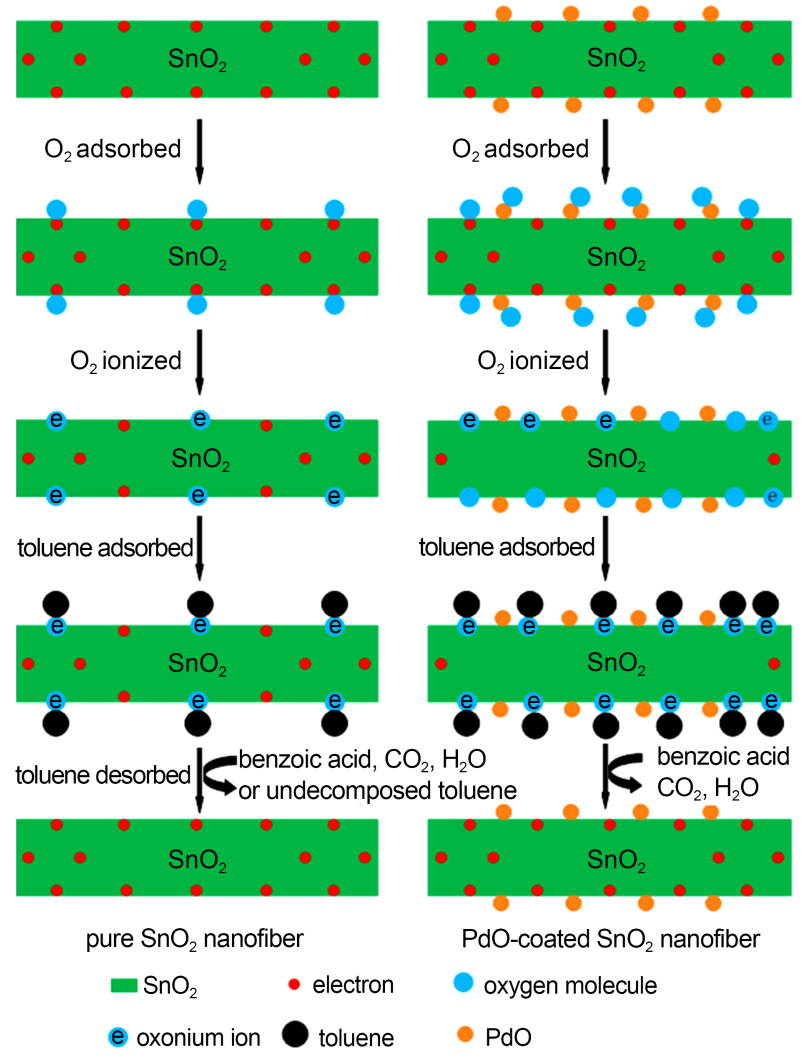

图10 纯 $\mathrm{SnO}_{2} 、 \mathrm{PdO}$ 修饰的 $\mathrm{SnO}_{2}$ 气敏元件对甲苯的气敏机 理模型

Fig.10 Schematic diagrams on the gas sensing mechanism of pure $\mathrm{SnO}_{2}$ and $\mathrm{PdO}$-coated $\mathrm{SnO}_{2}$ sensors to toluene

$\mathrm{O}_{2}+\mathrm{e}^{-} \rightarrow \mathrm{O}_{2}^{-}$(ads)

$\mathrm{O}_{2(\mathrm{ads})}^{-}+\mathrm{e}^{-} \rightarrow 2 \mathrm{O}^{-}{ }_{(\mathrm{ads})}$

$\mathrm{O}_{2(\text { ads })}^{-}+\mathrm{e}^{-} \rightarrow \mathrm{O}_{(\text {ads })}^{2-}$

$\mathrm{C}_{6} \mathrm{H}_{5} \mathrm{CH}_{3}+2 \mathrm{O}_{(\mathrm{ads})}^{-} \rightarrow \mathrm{C}_{6} \mathrm{H}_{5} \mathrm{CHO}+\mathrm{H}_{2} \mathrm{O}+2 \mathrm{e}^{-}$

$\mathrm{C}_{6} \mathrm{H}_{5} \mathrm{CHO}+16 \mathrm{O}_{(\text {ads })}^{-} \rightarrow 7 \mathrm{CO}_{2}+3 \mathrm{H}_{2} \mathrm{O}+16 \mathrm{e}^{-}$

纯 $\mathrm{SnO}_{2}$ 气敏元件对甲苯的响应很低, 在 300 ${ }^{\circ} \mathrm{C}$ 的工作温度下, 对 $30 \times 10^{-6}$ 甲苯的响应仅为 1.7 , 且 响应恢复时间较长, 分别为 120 和 $136 \mathrm{~s}$. 修饰PdO的 $\mathrm{SnO}_{2}$ 气敏元件对甲苯响应提高到 11.3, 响应恢复时 间也分别降低到 82 和 $63 \mathrm{~s}$. 可见PdO在气敏元件对甲
苯响应的提高中起到了非常重要的作用.

纯 $\mathrm{SnO}_{2}$ 气敏元件、 $\mathrm{PdO}$ 修饰的 $\mathrm{SnO}_{2}$ 气敏元件对 甲苯的气敏机理模型如图10所示. 修饰后气敏特性 增强可以从一下两个方面解释，一是用溢流效应： 由于 $\mathrm{O}_{2}$ 更容易在 $\mathrm{PdO}$ 的表面分解, ${ }^{27}$ 因此当将 $\mathrm{PdO}$ 粒 子负载在 $\mathrm{SnO}_{2}$ 表面后, 会有更多的 $\mathrm{O}_{2}$ 在 $\mathrm{PdO}$ 粒子表 面吸附并分解为氧原子, 氧原子迁移到 $\mathrm{SnO}_{2}$ 表面, 从 $\mathrm{SnO}_{2}$ 中俘获电子成为氧离子, 使得 $\mathrm{SnO}_{2}$ 表面会有 更多的氧离子参与反应, 故而使 $\mathrm{SnO}_{2}$ 材料的气敏响 应增强. 二是 $\mathrm{PdO}$ 作为一种催化剂, 对甲苯的分解有 催化作用, 使得甲苯更易于分解且分解的速率增快, 即反应(9)和(10)更易于进行. 这样会有更多的电子 被释放到材料中, $\mathrm{SnO}_{2}$ 材料电阻变化增大.

而 $\mathrm{Au}$ 修饰 $\mathrm{SnO}_{2}$ 对甲醛气敏特性的增强还与 $\mathrm{Au}$ 与 $\mathrm{SnO}_{2}$ 接触后, $\mathrm{Au}$ 从 $\mathrm{SnO}_{2}$ 表面夺取电子, 使得 $\mathrm{SnO}_{2}$ 的耗尽层加宽有关. 上文提到的纯 $\mathrm{SnO}_{2}$ 和 $\mathrm{Au}$ 修 饰的 $\mathrm{SnO}_{2}$ 的XPS表征中, $\mathrm{Sn}$ 峰位的移动, 证明了 $\mathrm{Au}$ 与 $\mathrm{Sn}$ 之间有相互作用, 且电子是从 $\mathrm{SnO}_{2}$ 流向 $\mathrm{Au} ;^{28}$ 二是修饰 $\mathrm{Au}$ 后, 材料的吸附氧含量增大, 即有更多 的氧离子参与反应, 增强气敏特性. 纯 $\mathrm{SnO}_{2}$ 的 $\mathrm{O}_{\text {adsorbed }} / \mathrm{Sn}$ 比为 0.58 , 而修饰 $\mathrm{Au}$ 后增加为 0.66 .

\section{4 结 论}

采用静电纺丝法制备了 $\mathrm{SnO}_{2}$ 纳米纤维材料, 该 $\mathrm{SnO}_{2}$ 材料为四方相金红石型结构, 纤维直径为 200 $\mathrm{nm}$ 左右, 且该纤维由大小为 $15 \mathrm{~nm}$ 的小颗粒堆积而 成. 对比不同物质修饰 $\mathrm{SnO}_{2}$ 的气敏特性, 结果表明, $\mathrm{CdO}$ 修饰的 $\mathrm{SnO}_{2}$ 气敏元件对甲醛的响应值的提高最 明显, 纯 $\mathrm{SnO}_{2}$ 气敏元件对 $10 \times 10^{-6}$ 甲醛的响应值为 5.6, 而 $\mathrm{CdO}$ 修饰的 $\mathrm{SnO}_{2}$ 气敏元件的响应值为 23.4 , 提 高了4倍多; 同时, $\mathrm{CdO}$ 修饰的 $\mathrm{SnO}_{2}$ 气敏元件的选择 性和稳定性最好. $\mathrm{Au}$ 修饰的 $\mathrm{SnO}_{2}$ 气敏元件则将纯 $\mathrm{SnO}_{2}$ 气敏元件的最佳工作温度由 $300{ }^{\circ} \mathrm{C}$ 降低为 200 ${ }^{\circ} \mathrm{C} . \mathrm{PdO}$ 修饰的 $\mathrm{SnO}_{2}$ 气敏元件则对甲苯的响应值最 高. 修饰后材料气敏特性的增强与溢流效应和修饰 
物的催化效应有关, 同时, 修饰物如 $\mathrm{Au}$ 与 $\mathrm{SnO}_{2}$ 接触 后从 $\mathrm{SnO}_{2}$ 表面夺取电子, 加宽其耗尽层也是修饰后 材料气敏特性增强的原因之一.

\section{References}

(1) Xu, J. Q.; Jia, X. H.; Lou, X. D.; Xi, G. X.; Han, J. J.; Gao, Q. H. Sens. Actuator B-Chem. 2007, 120, 694. doi: 10.1016/j.snb.2006.03.033

(2) Sun, P.; Zhou, X.; Wang, C.; Wang, B.; Xu, X.; Lu, G. Sens. Actuator B-Chem. 2014, 190, 32. doi: 10.1016/j.snb.2013.08.045

(3) Katoch, A.; Byun, J. H.; Choi, S. W.; Kim, S. S. Sens. Actuator B-Chem. 2014, 202, 38.

(4) Khoang, N. D.; Trung, D. D.; Van, D. N.; Hoa, N. D.; Van, H. N. Sens. Actuator B-Chem. 2012, 174, 594. doi: 10.1016/j.snb.2012.07.118

(5) Lin, Q.; Li, Y.; Yang, M. Sens. Actuator B-Chem. 2012, 173, 139. doi: 10.1016/j.snb.2012.06.055

(6) Sun, P.; Wang, C.; Zhou, X.; Cheng, P.; Shimanoe, K.; Lu, G.; Yamazoe, N. Sens. Actuator B-Chem. 2014, 193, 616. doi: 10.1016/j.snb.2013.12.015

(7) Mohanapriya, P.; Segawa, H.; Watanabe, K.; Watanabe, K.; Samitsu, S.; Natarajan, T. S.; Jaya, N. V.; Ohashi, N. Sens. Actuator B-Chem. 2013, 188, 872. doi: 10.1016/j.snb.2013.07.016

(8) Kaur, J.; Vankar, V. D.; Bhatnagar, M. C. Thin Solid Films 2010, 518, 3982. doi: 10.1016/j.tsf.2009.11.016

(9) Guan, Y.; Wang, D.; Zhou, X.; Sun, P.; Wang, H.; Ma, J.; Lu, G. Sens. Actuator B-Chem. 2014, 191, 45. doi: 10.1016/j.snb.2013.09.002

(10) Zheng, Y.; Wang, J.; Yao, P. Sens. Actuator B-Chem. 2011, 156, 723. doi: $10.1016 /$ j.snb.2011.02.026

(11) Zhang, F. H.; Wang, X. H.; Dong, J. P.; Qin, N.; Xu, J. Q. Sens. Actuator B-Chem. 2013, 186, 126. doi: 10.1016/j.snb.2013.05.086

(12) Hwang, I. S.; Choi, J. K.; Woo, H. S.; Kim, S. J.; Jung, S.; Seong, T. Y.; Kim, I. D.; Lee, J. H. ACS Appl. Mater. Inter. 2011, 3, 3140. doi: 10.1021/am200647f

(13) Zheng, K. B.; Li, J. L.; Shen, H. T.; Sun, D. L.; Chen, G. R. Acta Phys. -Chim. Sin. 2008, 24, 1080. [郑凯波, 李静雷, 沈浩频, 孙 大林, 陈国荣. 物理化学学报, 2008, 24, 1080.] doi 10.3866/PKU.WHXB20080629

(14) Chen, P. P.; Wang, J.; Zhang, C. L.; Hao, Y. W.; Du, H. Y. Acta
Phys. -Chim. Sin. 2013, 29, 1827. [陈鹏鹏, 王 兢, 张春丽, 郝 育闻, 杜海英. 物理化学学报, 2013, 29, 1827.] doi: 10.3866/PKU.WHXB201306091

(15) Zhao, M.; Wang, J. X.; Feng, C. H.; Zou, B.; Chen, C.; Wang, Z. Y.; Wu, F. Q.; Zou, L. H. Acta Phys. -Chim. Sin. 2007, 23, 1003. [赵 萌, 王金兴, 冯彩慧, 邹 博, 陈 骋, 王竹仪, 吴风清, 邹 乐辉. 物理化学学报, 2007, 23, 1003.] doi: 10.3866/PKU.WHXB20070708

(16) Jin, C.; Kim, H.; An, S.; Lee, C. Ceram. Int. 2012, 38, 5973. doi: 10.1016/j.ceramint.2012.04.050

(17) Jain, G. H.; Patil, L. A.; Wagh, M. S.; Patil, D. R.; Patil, S. A.; Amalnerkar, D. P. Sens. Actuator B-Chem. 2006, 117, 159. doi: 10.1016/j.snb.2005.11.031

(18) He, J. Q.; Yin, J.; Liu, D.; Zhang, L. X.; Cai, F. S.; Bie, L. J. Sens. Actuator B-Chem. 2013, 182, 170. doi: 10.1016/j.snb.2013.02.085

(19) Cao, H. Q.; Zhu, M. F.; Li, Y. G. J. Solid State Chem. 2006, 179, 1208. doi: 10.1016/j.jssc.2005.12.040

(20) Vuong, N. M.; Hieu, N. M.; Hieu, H..; Yi, H.; Kim, D.; Han, Y. S.; Kim, M. Sens. Actuator B-Chem. 2014, 192, 327. doi: 10.1016/j.snb.2013.10.117

(21) Liang, Y. C.; Liao, W. K.; Deng, X. S. J. Alloy. Compd. 2014, 599, 87. doi: 10.1016/j.jallcom.2014.01.167

(22) Wang, B.; Zheng, Z. Q.; Zhu, L. F.; Yang, Y. H.; Wu, H. Y. Sens. Actuator B-Chem. 2014, 195, 549. doi: 10.1016/j.snb.2014.01.073

(23) Zheng, W.; Lu, X.; Wang, W.; Li, Z.; Zhang, H.; Wang, Z.; Xu, X.; Li, S.; Wang, C. J. Colloid Interface Sci. 2009, 338, 366. doi: 10.1016/j.jcis.2009.06.041

(24) Sun, L.; Li, J.; Wang, C.; Li, S.; Lai, Y.; Chen, H.; Lin, C. J. Hazard. Mater. 2009, 171, 1045. doi: 10.1016/j.jhazmat.2009.06.115

(25) Liu, X.; Zhang, J.; Yang, T.; Guo, X.; Wu, S.; Wang, S. Sens. Actuator B-Chem. 2011, 156, 91.

(26) Vaishnav, V. S.; Patel, S. G.; Panchal, J. N. Sens. Actuator BChem. 2015, 210, 165. doi: 10.1016/j.snb.2014.11.075

(27) Kolmakov, A.; Klenov, D. O.; Lilach, Y.; Stemmer, S.; Moskovits, M. Nano Letters 2005, 5, 667. doi: $10.1021 / \mathrm{n} 1050082 \mathrm{v}$

(28) Wang, L.; Kang, Y.; Wang, Y.; Zhu, B.; Zhang, S.; Huang, W.; Wang, S. Mat. Sci. Eng. C 2012, 32, 2079. doi: 10.1016/j.msec.2012.05.042 